\title{
An Efficient Class of Modulus-Based Matrix Splitting Methods for Nonlinear Complementarity Problems
}

\author{
Shui-Lian Xie $(i)$ and Hong-Ru Xu (iD \\ School of Mathematics, Jiaying University, Meizhou, Guangdong 514015, China \\ Correspondence should be addressed to Hong-Ru Xu; hrxu001@163.com
}

Received 5 October 2021; Revised 12 December 2021; Accepted 14 December 2021; Published 28 December 2021

Academic Editor: Fangqing Wen

Copyright (c) 2021 Shui-Lian Xie and Hong-Ru Xu. This is an open access article distributed under the Creative Commons Attribution License, which permits unrestricted use, distribution, and reproduction in any medium, provided the original work is properly cited.

In this paper, we present an efficient method for finding a numerical solution for nonlinear complementarity problems (NCPs). We first reformulate an NCP as an equivalent system of fixed-point equations and then present a modulus-based matrix splitting iteration method. We prove the convergence of the proposed method with theorems with the relevant conditions. Our preliminary numerical results show that the method is feasible and effective.

\section{Introduction}

In this paper, we consider the following nonlinear complementarity problem with a nonlinear source term, namely, finding a $u \in R^{n}$ such that

$$
\begin{aligned}
u & \geq 0, \\
v & =F(u) \geq 0, \\
u^{T} v & =0,
\end{aligned}
$$

where the function $F(u)$ has the form of $A u+\Phi(u)+q, A=$ $\left(a_{i j}\right) \in R^{n \times n}$ is a given large, sparse, and real matrix, $q$ is a given vector, $\Phi: R^{n} \longrightarrow R^{n}$ is a given diagonal differentiable mapping, that is, the $i$ th component $\Phi_{i}$ of $\Phi$ is a function of the $i$ th variable $u_{i}$ only

$$
\Phi_{i}=\Phi_{i}\left(u_{i}\right), \quad i=1,2, \ldots, n .
$$

Clearly, if $\Phi$ is a linear function, problem (1) reduces to a linear complementarity problem (LCP).

Nonlinear complementarity problem (NCP) has many applications in nonlinear compressed sensing, signal processing, communications, DNA microarrays, and so on [1-7]. For example, the nonlinear compressed sensing theory has been widely applied in asteroseimology for significant detection. The polynomial structure has been employed in many applications cases, such as quadratic measurements in sparse signal recovery and nonlinear compressed sensing with polynomial measurements. In this regard, under some constraint qualifications, the original problem turns out to finding the sparsest solutions to a special NCP. NCP can also be derived from discrete simulations of the Bratu obstacle problem and free boundary problems with nonlinear source terms. This problem has received much attention during the past two decades and has been studied extensively with applicable numerical methods to obtain an approximated solution. There are different kinds of numerical methods that have been developed, including the classical linearized projected relaxation method [8], multilevel method [9], domain decomposition method $[10,11]$, penalty method [12-14], and semismooth Newton method [15]. Most of those methods need to solve the linear complementarity subproblems; see [16-19] for several typical iteration methods.

It is well known that the matrix splitting method is a very important and effective method for computing systems of linear equations. Many authors have extended this kind of method for LCP. Bai [20] presented a unified framework for 
the construction of modulus-based matrix splitting iteration methods. Since then, many scholars have developed various kinds of modulus-based matrix splitting iteration methods; see, for example, [21-23] for LCP and [24, 25] for NCP. Recently, Wu and Li [26] proposed a class of new modulusbased matrix splitting methods for LCP. This kind of method is different from previously published works. The new modulus-based matrix splitting iteration methods provide a new general framework for solving the LCP. Numerical experiments show that the new modulus-based matrix splitting iteration method for LCP is feasible and competitive when compared with other well-known modulus-based matrix splitting iteration methods. Motivated by the ongoing work in this field, in this paper, we extend the new modulusbased matrix splitting method for a kind of NCP and establish its convergence through theorems.

The remainder of this paper is structured as follows. In Section 2, we present some relevant preliminary information and the modulus-based matrix splitting iteration method. We establish the convergence theorems of the method under suitable conditions in Section 3. In Section 4, we present numerical results to show the efficiency of the proposed method.

\section{Modulus-Based Matrix Splitting Iteration Method}

We briefly introduce some definitions and lemmas that will be used in the paper. Most of them can be found in $[20,25,27,28]$.

Given two matrices $A=\left(a_{i j}\right), B=\left(b_{i j}\right) \in R^{n \times n}$, we call $A \geq B(A>B)$ if $a_{i j} \geq b_{i j}\left(a_{i j}>b_{i j}\right)$ holds for all $1 \leq i \leq n$, $1 \leq j \leq n$. If $a_{i j} \geq 0$ for all $1 \leq i \leq n, 1 \leq j \leq n, A$ is called a nonnegative matrix, denoted by $A \geq O$. $|A|$ denotes the nonnegative matrix with entries $\left|a_{i j}\right|$.

Let $A$ be a real $n \times n$ matrix. Its comparison matrix $\langle A\rangle=\left(\left\langle a_{i j}\right\rangle\right) \in R^{n \times n}$, where

$$
\left\langle a_{i j}\right\rangle=\left\{\begin{array}{ll}
\left|a_{i j}\right|, & i=j \\
-\left|a_{i j}\right| x, & i \neq j
\end{array} \quad i, j=1,2, \ldots, n .\right.
$$

The matrix $A$ is called a $Z$-matrix if its off-diagonal entries are all nonpositive; $A$ is called an $M$-matrix if $A$ is a $Z$-matrix and $A^{-1} \geq O ; A$ is called an $H$-matrix if its comparison matrix $\langle A\rangle$ is an $M$-matrix; $A$ is called an $\mathrm{H}_{+}$-matrix if it is an $\mathrm{H}$-matrix with positive diagonal entries; if $A$ is an $M$-matrix, $\Omega$ is a positive diagonal matrix, and then $A \leq B \leq \Omega$ implies that $B$ is an $M$ - matrix. We use $\rho(A)$ to represent the spectral radius of the square matrix $A \in R^{n \times n}$

Given $A=M-N$, if $M$ is a nonsingular matrix, then $A=M-N$ is called a splitting of the matrix $A$; for the splitting $A=M-N$, if $\langle M\rangle-|N|$ is an $M$-matrix, the splitting is called a $H$-splitting.

Lemma 1 (see [27]). Let $A=\left(a_{i j}\right) \in R^{n \times n}$ with $a_{i j} \geq 0$. If there exists $u \in R^{n}$ with $u>0$ such that $A u<u$, then $\rho(A)<1$.
Lemma 2 (see [28]). Let $A \in R^{n \times n}$ be an $M$-matrix and $B \in R^{n \times n}$ be a $Z$ - matrix with $A \leq B$. Then, $B$ is an $M$-matrix.

Lemma 3 (see [28]). Let $A \in R^{n \times n}$ be an $H$-matrix, $\widehat{D}$ be the diagonal part of the matrix $A$, and $A=\widehat{D}-B$. Then, matrices $A$ and $|\widehat{D}|$ are nonsingular, $\left|A^{-1}\right| \leq\langle A\rangle^{-1}$ and $\rho\left(|\widehat{D}|^{-1}|B|\right)<1$.

Lemma 4 (see [26]). Let $A=M-N$ be an $H$-splitting of the matrix $A$, where $A$ is an $H_{+}-$matrix. Then, $\langle M\rangle-|N| \leq \widehat{D}-|B|$, where $\widehat{D}=\operatorname{diag}(A)$ and $A=\widehat{D}-B$.

To obtain a new equivalence system of fixed-point equations of the NCP, we need the following lemma:

Lemma 5 (see [26]). Let $a, b \in R$. Then,

$$
a \geq 0, b \geq 0, a b=0,
$$

if and only if

$$
a+b=|a-b|
$$

This result carries immediately over to the vectors in $R^{n}$. Based on Lemma 5, we have the following theorem:

Theorem 1. Let $\Omega$ be a positive diagonal matrix. Then, NCP (1.1) is equal to

$$
(\Omega+A) u=|(A-\Omega) u+\Phi(u)+q|-q-\Phi(u) .
$$

Proof. Obviously, NCP (1.1) is equal to

$$
v=A u+\Phi(u)+q \geq 0, \quad \Omega u \geq 0,(\Omega u)^{T} v=0,
$$

where $\Omega$ is a positive diagonal matrix. Here, we take $a=$ $A u+\Phi(u)+q$ and $b=\Omega u$ for Lemma 5 and get

$$
(\Omega+A) u=|(A-\Omega) u+\Phi(u)+q|-q-\Phi(u) .
$$

The proof is completed.

We take $A=M-N$ as a matrix splitting of matrix $A \in R^{n \times n}$; then, from (6), we have

$$
(\Omega+M) u=N u+|(A-\Omega) u+\Phi(u)+q|-q-\Phi(u) .
$$

This new equivalent expression (9) is different from the one in the previous work in $[24,25]$. These methods have similarities but they do not belong to each other. Based on (9), we can present the following modulus-based matrix splitting iteration method for solving (1).

Method 1 (modulus-based matrix splitting iteration method). Let $A=M-N$ be a splitting of the matrix $A \in R^{n \times n}$ and $M+\Omega$ be a nonsingular matrix, where $\Omega$ is a positive diagonal matrix. Given an initial vector $u^{0} \in R^{n}$, for $k=0,1,2, \cdots$ until the iteration sequence $\left\{u^{k}\right\}_{k=0}^{\infty} \subset R^{n}$ is convergent, compute $u^{k+1} \in R^{n}$ by

$$
(\Omega+M) u^{k+1}=N u^{k}+\left|(A-\Omega) u^{k}+\Phi\left(u^{k}\right)+q\right|-q-\Phi\left(u^{k}\right) .
$$


Method 1 provides a general framework of the modulusbased matrix splitting iteration method for solving the NCP. Based on the matrix splitting technique, some new modulusbased relaxation methods are obtained. For example, we express matrix $A$ as

$$
A=\widehat{D}-L-U
$$

where $\widehat{D}=\operatorname{diag}(A)$ and $-L$ and $-U$ are the strictly lower and upper triangular matrices of $A$, respectively. Then,

(i) When $M=A, \Omega=I, N=0$, from Method 1, we have

$$
(I+A) u^{k+1}=\left|(A-I) u^{k}+\Phi\left(u^{k}\right)+q\right|-q-\Phi\left(u^{k}\right)
$$

which is called a modulus iteration (MI) method.

(ii) When

$$
M=1 / \alpha(\widehat{D}-\beta L)
$$

$N=1 / \alpha((1-\alpha) \widehat{D}+(\alpha-\beta) L+\alpha U)$, from Method 1 , we have

$$
\begin{aligned}
(\alpha \Omega+\widehat{D}-\beta L) u^{k+1}= & ((1-\alpha) \widehat{D}+(\alpha-\beta) L+\alpha U) u^{k} \\
& +\alpha\left|(A-\Omega) u^{k}+\Phi\left(u^{k}\right)+q\right|-q-\Phi\left(u^{k}\right),
\end{aligned}
$$

which is called the modulus-based AOR (MAOR) iteration method. Clearly, the MAOR method becomes the new modulus-based successive overrelaxation, Gauss-Seidel, and Jacobi method when $(\alpha, \beta)$ is equal to $(\alpha, \alpha),(1,1)$, and $(1,0)$, respectively.

\section{Convergence Theory}

In this section, we discuss the convergence of Method 1 under suitable conditions.

Theorem 2. Let $\Omega$ be an $n \times n$ positive diagonal matrix, $A=$ $M-N$ be a splitting of a matrix $A \in R^{n \times n}$, with $M+\Omega$ being a nonsingular matrix. Suppose that there exist positive constants $d_{i}$ such that $d_{i} \geq d \Phi_{i}\left(u_{i}\right) / d u_{i} \geq 0, i=1,2, \ldots, n$, for all $u \in R^{n}$. Let $D$ be a diagonal matrix, and the diagonal elements are $d_{i}, i=1,2, \ldots, n$. Let

$$
T=\left|(\Omega+M)^{-1}\right|(|N|+|A-\Omega|+2 D) .
$$

If $\rho(T)<1$, then the iteration sequence $\left\{u^{k}\right\}_{k=0}^{+\infty} \subset R^{n}$ generated by Method 1 converges to the solution $u^{\star}$ of NCP (1.1).

Proof. Let $u^{\star}$ be the solution for the NCP (1.1). Then, from (6), we obtain

$$
(\Omega+M) u^{\star}=N u^{\star}+\left|(A-\Omega) u^{\star}+\Phi\left(u^{\star}\right)+q\right|-q-\Phi\left(u^{\star}\right) .
$$

Based on (10) and (15), noting that $\Omega+M$ is nonsingular, we have

$$
u^{k+1}-u^{\star}=(M+\Omega)^{-1}\left[N\left(u^{k}-u^{\star}\right)+\Phi\left(u^{\star}\right)-\Phi\left(u^{k}\right)+\left|(A-\Omega) u^{k}+\Phi\left(u^{k}\right)+q\right|-\left|(A-\Omega) u^{\star}+\Phi\left(u^{\star}\right)+q\right|\right]
$$

This indicates that

$$
\begin{aligned}
\left|u^{k+1}-u^{\star}\right|= & \left|(M+\Omega)^{-1}\left[N\left(u^{k}-u^{\star}\right)+\Phi\left(u^{\star}\right)-\Phi\left(u^{k}\right)+\left|(A-\Omega) u^{k}+\Phi\left(u^{k}\right)+q\right|-\left|(A-\Omega) u^{\star}+\Phi\left(u^{\star}\right)+q\right|\right]\right| \\
\leq & \left|(M+\Omega)^{-1} N\left(u^{k}-u^{\star}\right)\right|+\left|(M+\Omega)^{-1}\right| \cdot\left|\Phi\left(u^{\star}\right)-\Phi\left(u^{k}\right)\right| \\
& +\left|(M+\Omega)^{-1}\right| \cdot||(A-\Omega) u^{k}+\Phi\left(u^{k}\right)+q|-|(A-\Omega) u^{\star}+\Phi\left(u^{\star}\right)+q|| \\
\leq & \left|(M+\Omega)^{-1} N\right| \cdot\left|u^{k}-u^{\star}\right|+\left|(M+\Omega)^{-1}\right| D\left|u^{k}-u^{\star}\right|+\left|(M+\Omega)^{-1}\right|(|A-\Omega|+D)\left|u^{k}-u^{\star}\right| \\
= & \left|(M+\Omega)^{-1}\right|(|N|+|A-\Omega|+2 D)\left|u^{k}-u^{\star}\right| .
\end{aligned}
$$


It is clear that when $\rho(T)<1$, the iteration sequence $\left\{u^{k}\right\}_{k=0}^{+\infty} \subset R^{n}$ generated by Method 1 converges to the solution $u^{\star}$ of NCP (1.1). This completes the proof.

Similar to the proof of Theorem 2, using $\|\cdot\|_{2}$ for (3.2), we have the following theorem.

Theorem 3. Let $\Omega$ be an $n \times n$ positive diagonal matrix, let $A=M-N$ be a splitting of a matrix $A \in R^{n \times n}$, with $M+\Omega$ being a nonsingular matrix. Suppose that there exist positive constants $d_{i}$ such that $d_{i} \geq d \Phi_{i}\left(u_{i}\right) / d u_{i} \geq 0, i=1,2, \ldots, n$, for all $u \in R^{n}$. Let $D$ be a diagonal matrix, and the diagonal elements are $d_{i}, i=1,2, \ldots, n$. Let

$$
\widehat{T}=\left\|(\Omega+M)^{-1}\right\|_{2}\left(\|N\|_{2}+\|A-\Omega\|_{2}+\|2 D\|_{2}\right) .
$$

If $\rho(\widehat{T})<1$, then the iteration sequence $\left\{u^{k}\right\}_{k=0}^{+\infty} \subset R^{n}$ generated by Method 1 converges to the solution $u^{\star}$ of the NCP (1.1).
Theorem 4. Let $A=M-N$ be an $H$-splitting of the matrix $A$, where $A$ is an $H_{+}-$matrix. Let the diagonal matrix $\Omega \geq \widehat{D}$, where $\widehat{D}=\operatorname{diag}(A)$. Suppose there exist positive constants $d_{i}$ such that $d_{i} \geq d \Phi_{i}\left(u_{i}\right) / d u_{i} \geq 0, i=1,2, \ldots, n$, for all $u \in R^{n}$. Let $D$ be a diagonal matrix, and the diagonal elements are $d_{i}$, $i=1,2, \ldots, n$. Let $T=\left|(\Omega+M)^{-1}\right|(|N|+|A-\Omega|+2 D)$. If $\langle M\rangle-|N|-D$ is an $M-$ matrix, then the iteration sequence $\left\{u^{k}\right\}_{k=0}^{+\infty} \subset R^{n}$ generated by Method 1 converges to the solution $u^{\star}$ of the NCP (1.1).

Proof. Since $A=M-N$ is an $H$-splitting and

$$
\langle M\rangle-|N| \leq\langle M\rangle \text {. }
$$

Matrix $\langle M\rangle$ is an $M$-matrix from Lemma Method 2. Noting that $\Omega \geq \widehat{D}$, by Lemma 3 , we can see $\Omega+M$ is an $H_{+}-$matrix and

$$
\left|(M+\Omega)^{-1}\right| \leq(\langle M\rangle+\Omega)^{-1} .
$$

Moreover, we have

$$
\begin{aligned}
T & \leq(\langle M\rangle+\Omega)^{-1}(|N|+|A-\Omega|+2 D) \\
& =(\langle M\rangle+\Omega)^{-1}(\langle M\rangle+\Omega-\langle M\rangle-\Omega+|N|+|A-\Omega|+2 D) \\
& \leq(\langle M\rangle+\Omega)^{-1}(\langle M\rangle+\Omega-\langle M\rangle-\Omega+|N|+|\widehat{D}-\Omega|+|B|+2 D) \\
& =I-(\langle M\rangle+\Omega)^{-1}(\langle M\rangle+\Omega-\langle M\rangle-\Omega-|\widehat{D}-\Omega|-|B|-2 D) \\
& =I-(\langle M\rangle+\Omega)^{-1}(\langle M\rangle-|N|+\widehat{D}-|B|-2 D) \\
& \leq I-2(\langle M\rangle+\Omega)^{-1}(\langle M\rangle-|N|-D),
\end{aligned}
$$

where the last inequality holds by Lemma 4 . Since $\langle M\rangle-$ $|N|-D$ is an $M$-matrix, there exists a positive vector $u$ such that

$$
(\langle M\rangle-|N|-D) u>0
$$

Hence,

$$
T u \leq\left(I-2(\langle M\rangle+\Omega)^{-1}(\langle M\rangle-|N|-D)\right) u<u .
$$

We have $\rho(T)<1$ by Lemma 1 , and the iteration sequence $\left\{u^{k}\right\}_{k=0}^{+\infty} \subset R^{n}$ generated by Method 1 converges to the solution of NCP (1.1). This completes the proof.

\section{Numerical Experiments}

In this section, we test the effectiveness of the proposed method on two problems. We implemented our methods in MATLAB and ran the code on a PC with a $3.4 \mathrm{GHz} C P U$ and 8GB RAM. We tested the proposed method in Section 2 and compared their performance with Method 4.1 proposed in [24].

Method 2. Let $A=M-N$ be a splitting of the matrix $A \in R^{n \times n}, \Omega$ be an $n \times n$ nonnegative diagonal matrix, and $h$ be a positive number. Given $x^{0} \in R^{n}$, for $k=0,1,2, \cdots$, compute $x^{k+1} \in R^{n}$ by solving the linear system:

$$
\begin{aligned}
(\Omega+M) x^{k+1}= & N x^{k}+(\Omega-A)\left|x^{k}\right| \\
& -\frac{2}{h}\left(q+\Phi\left(\frac{h}{2}\left(\left|x^{k}\right|+x^{k}\right)\right)\right)
\end{aligned}
$$

and set

$$
u^{k+1}=\frac{h}{2}\left(\left|x^{k+1}\right|+x^{k+1}\right)
$$

The iteration terminates when the sequence $\left\{x^{k}\right\}_{k=0}^{\infty} \subset R^{n}$ is convergent. 
TABLE 1: Numerical results of NMI for Problem 1 with different $\Omega$.

\begin{tabular}{lccccc}
\hline & $\Omega=I$ & $\Omega=2 I$ & $\Omega=3 I$ & $\Omega=4 I$ & $\Omega=5 I$ \\
\hline CPU & 3.254 & 1.128 & 0.802 & 0.522 & 0.644 \\
Iter & 87 & 29 & 21 & 13 & 17 \\
Res & $8.8485 e-7$ & $7.1188 e-7$ & $5.6296 e-7$ & $9.7839 e-7$ & $4.8359 e-7$ \\
\hline
\end{tabular}

TABle 2: Numerical results of NMSOR for Problem 1 with different $\Omega$.

\begin{tabular}{lccccc}
\hline & $\Omega=I$ & $\Omega=2 I$ & $\Omega=3 I$ & $\Omega=4 I$ & $\Omega=5 I$ \\
\hline CPU & 1.812 & 0.913 & 0.804 & 0.779 & 21 \\
Iter & 50 & 25 & 22 & 26 & 265 \\
Res & $8.8387 e-7$ & $9.0549 e-7$ & $8.2116 e-7$ & $6.6182 e-7$ & $9.1418 e-7$ \\
\hline
\end{tabular}

Table 3: Numerical results for Problem 1 using Method 2.

\begin{tabular}{|c|c|c|c|c|c|c|c|}
\hline$n$ & & MI & $\mathrm{MSOR}_{0.2}$ & $\mathrm{MSOR}_{0.4}$ & $\mathrm{MSOR}_{0.6}$ & $\mathrm{MSOR}_{0.8}$ & $\mathrm{MSOR}_{1.0}$ \\
\hline \multirow{3}{*}{100} & $\mathrm{CPU}$ & 0.1 & 0.121 & 0.054 & 0.036 & 0.056 & 0.37 \\
\hline & Iter & 86 & 121 & 60 & 41 & 64 & 447 \\
\hline & Res & $9.7893 e-7$ & $9.5296 e-7$ & $8.8894 e-7$ & $9.3262 e-7$ & $9.5452 e-7$ & $9.6319 e-7$ \\
\hline \multirow{3}{*}{400} & $\mathrm{CPU}$ & 3.35 & 4.778 & 2.319 & 1.616 & 3.029 & - \\
\hline & Iter & 87 & 127 & 62 & 43 & 81 & - \\
\hline & Res & $8.9383 e-7$ & $9.9707 e-7$ & $9.0364 e-7$ & $7.7447 e-7$ & $8.7010 e-7$ & - \\
\hline \multirow{3}{*}{900} & $\mathrm{CPU}$ & 34.022 & 50.732 & 24.375 & 16.623 & 34.383 & - \\
\hline & Iter & 87 & 131 & 63 & 43 & 89 & - \\
\hline & Res & $9.3875 e-7$ & $9.5828 e-7$ & $9.4402 e-7$ & $9.5916 e-7$ & $8.6957 e-7$ & - \\
\hline \multirow{3}{*}{1600} & $\mathrm{CPU}$ & 188.867 & 288.017 & 136.851 & 94.189 & 192.049 & - \\
\hline & Iter & 87 & 134 & 64 & 44 & 90 & - \\
\hline & Res & $9.8162 e-7$ & $9.123 e-7$ & $9.1586 e-7$ & $8.382 e-7$ & $8.8297 e-7$ & - \\
\hline
\end{tabular}

In all the experiments, we chose the same $\Omega$ and $h$ in Method 2 as in [24], that is, $\Omega=I$ and $h=1$.

We considered the following two problems:

Problem 1. Consider (1.1), let $F(u)=A u+\Phi(u)+q$, where $A=\left(\begin{array}{cccc}H & -I & & \\ -I & H & \ddots & \\ & \ddots & \ddots & -I \\ & & -I & H\end{array}\right)$ with $H=\left(\begin{array}{cccc}4 & -1 & & \\ -1 & 4 & \ddots & \\ & \ddots & \ddots & -1 \\ & & -1 & 4\end{array}\right)$,

$$
A=\left(\begin{array}{cccc}
H & -0.5 I & & \\
-1.5 I & H & \ddots & \\
& \ddots & \ddots & -0.5 I \\
& & -1.5 I & H
\end{array}\right)
$$

with $H=\left(\begin{array}{cccc}4 & -0.5 & & \\ -1.5 & 4 & \ddots & \\ & \ddots & \ddots & -0.5 \\ & & -1.5 & 4\end{array}\right)$,

$A$ is an $n \times n$ matrix, $H$ and $I$ are $t \times t$ matrices with $n=t^{2}$, and $\Phi_{i}\left(u_{i}\right)=\arctan \left(u_{i}\right)$ and a diagonal mapping $\Phi(u)=\left(\Phi_{i}\left(u_{i}\right)\right)$, and $q=(1,-1,1,-1, \ldots)^{T}$.

For the above problems, the initial points of all the methods were set to $u^{0}=(1,0,1,0, \ldots)^{T}$. Define Res :=
$A$ is an $n \times n$ matrix, $H$ and $I$ are $t \times t$ matrices with $n=t^{2}$, $\Phi_{i}\left(u_{i}\right)=u_{i} /\left(1+u_{i}\right)$ and a diagonal mapping $\Phi(u)=\left(\Phi_{i}\left(u_{i}\right)\right)$ and $q=(1-1,1,-1, \ldots)^{T}$.

Problem 2. Consider (1.1), let $F(u)=A u+\Phi(u)+q$, where $\left\|\min \left(u^{k}, F\left(u^{k}\right)\right)\right\|_{2}$ and the stopping criterion for all the methods are set to Res $<10^{-6}$. We can obtain many matrix splitting iteration methods from Methods 1 and 2 using two splittings of matrix $A=M-N$ : different splittings of $A$. In our experiments, we consider 
Table 4: Numerical results for Problem 1 using Method 1.

\begin{tabular}{ccccccccccc}
\hline$n$ & & NMI & NMSOR $_{0.2}$ & NMSOR $_{0.4}$ & NMSOR $_{0.6}$ & NMSOR $_{0.8}$ & NMSOR $_{1.0}$ & NMSOR $_{1.2}$ NMSOR $_{1.4}$ \\
\hline \multirow{4}{*}{100} & CPU & 0.027 & 0.074 & 0.027 & 0.017 & 0.012 & 0.014 & 0.017 \\
& Iter & 13 & 64 & 31 & 20 & 15 & 16 & 20 & 26 \\
& Res & $4.2714 e-7$ & $9.3221 e-7$ & $7.4581 e-7$ & $5.5552 e-7$ & $3.3274 e-7$ & $3.5144 e-7$ & $8.1510 e-7$ & $5.387 e-7$ \\
\hline \multirow{3}{*}{400} & CPU & 0.494 & 2.522 & 1.195 & 0.767 & 0.578 & 0.576 & 0.788 & 0.943 \\
& Iter & 13 & 69 & 33 & 21 & 16 & 16 & 21 & 26 \\
& Res & $9.7839 e-7$ & $8.2406 e-7$ & $8.7411 e-7$ & $6.6182 e-7$ & $3.2473 e-7$ & $5.2506 e-7$ & $5.832 e-7$ & $9.8892 e-7$ \\
\hline \multirow{3}{*}{900} & CPU & 5.747 & 26.628 & 12.643 & 8.189 & 5.956 & 5.952 & 7.824 & 10.072 \\
& Iter & 15 & 71 & 34 & 22 & 16 & 16 & 21 \\
& Res & $2.0204 e-7$ & $8.2307 e-7$ & $8.4129 e-7$ & $4.0343 e-7$ & $4.6232 e-7$ & $6.3577 e-7$ & $6.9295 e-7$ & $6.3989 e-7$ \\
\hline \multirow{2}{*}{1600} & CPU & 30.761 & 145.854 & 70.692 & 44.517 & 32.516 & 32.537 & 42.456 & 54.465 \\
& Iter & 15 & 72 & 35 & 22 & 16 & 16 & 21 \\
& Res & $2.6947 e-7$ & $8.9048 e-7$ & $6.624 e-7$ & $5.7269 e-7$ & $5.7292 e-7$ & $7.2981 e-7$ & $7.8753 e-7$ & $7.2316 e-7$ \\
\hline
\end{tabular}

TABle 5: Numerical results for Problem 2 using Method 2.

\begin{tabular}{|c|c|c|c|c|c|c|c|}
\hline$n$ & & MI & $\mathrm{MSOR}_{0.2}$ & $\mathrm{MSOR}_{0.4}$ & $\mathrm{MSOR}_{0.6}$ & $\mathrm{MSOR}_{0.8}$ & $\mathrm{MSOR}_{1.0}$ \\
\hline \multirow{3}{*}{100} & $\mathrm{CPU}$ & 0.685 & 0.129 & 0.055 & 0.038 & 0.058 & - \\
\hline & Iter & 700 & 121 & 63 & 44 & 67 & - \\
\hline & Res & $9.8494 e-7$ & $9.2832 e-7$ & $8.5078 e-7$ & $8.9841 e-7$ & $8.9822 e-7$ & - \\
\hline \multirow{3}{*}{400} & $\mathrm{CPU}$ & 29.496 & 4.735 & 2.424 & 1.682 & 2.538 & - \\
\hline & Iter & 699 & 126 & 65 & 45 & 68 & - \\
\hline & Res & $9.9211 e-7$ & $9.5234 e-7$ & $8.3278 e-7$ & $9.6855 e-7$ & $9.4709 e-7$ & - \\
\hline \multirow{3}{*}{900} & $\mathrm{CPU}$ & 275.014 & 50.976 & 26.225 & 18.25 & 26.826 & - \\
\hline & Iter & 699 & 129 & 66 & 46 & 68 & - \\
\hline & Res & $9.9235 e-7$ & $9.6376 e-7$ & $8.47 e-7$ & $9.0547 e-7$ & $9.4944 e-7$ & - \\
\hline \multirow{3}{*}{1600} & $\mathrm{CPU}$ & 1516.268 & 279.358 & 140.491 & 100.07 & 145.021 & - \\
\hline & Iter & 699 & 131 & 66 & 47 & 68 & - \\
\hline & Res & $9.9258 e-7$ & $9.895 e-7$ & $9.793 e-7$ & $9.9849 e-7$ & $9.5129 e-7$ & - \\
\hline
\end{tabular}

TABLe 6: Numerical results for Problem 2 using Method 1.

\begin{tabular}{ccccccccccc}
\hline$n$ & & NMI & NMSOR $_{0.2}$ & NMSOR $_{0.4}$ & NMSOR $_{0.6}$ & NMSOR $_{0.8}$ & NMSOR $_{1.0}$ & NMSOR $_{1.2}$ & NMSOR $_{1.4}$ \\
\hline \multirow{4}{*}{100} & CPU & 0.012 & 0.056 & 0.021 & 0.014 & 0.013 & 0.012 & 0.017 \\
& Iter & 15 & 52 & 25 & 16 & 12 & 14 & 19 & 24 \\
& Res & $4.1947 e-7$ & $9.3799 e-7$ & $9.2101 e-7$ & $8.1471 e-7$ & $9.399 e-7$ & $7.3949 e-7$ & $6.1808 e-7$ & $9.2176 e-7$ \\
\hline \multirow{3}{*}{400} & CPU & 0.595 & 2.021 & 0942 & 0.612 & 0.469 & 0.542 & 0.684 & 0.9 \\
& Iter & 15 & 55 & 26 & 17 & 13 & 15 & 19 & 25 \\
& Res & $9.384 e-7$ & $9.0552 e-7$ & $9.849 e-7$ & $6.0963 e-7$ & $5.9889 e-7$ & $4.7898 e-7$ & $7.7494 e-7$ & $6.3067 e-7$ \\
\hline \multirow{3}{*}{900} & CPU & 6.415 & 22.27 & 10.681 & 6.728 & 5.147 & 5.913 & 7.522 & 9.904 \\
& Iter & 16 & 56 & 27 & 17 & 13 & 15 & 19 & 25 \\
& Res & $2.1765 e-7$ & $9.86 e-7$ & $7.0764 e-7$ & $8.6029 e-7$ & $8.7608 e-7$ & $6.264 e-7$ & $8.22794 e-7$ & $6.9569 e-7$ \\
\hline \multirow{2}{*}{1600} & CPU & 34.508 & 115.098 & 54.534 & 36.327 & 28.291 & 30.261 & 38.417 & 50.468 \\
& Iter & 16 & 57 & 27 & 18 & 14 & 15 & 19 & 25 \\
& Res & $3.7312 e-7$ & $9.2141 e-7$ & $8.9017 e-7$ & $3.6077 e-7$ & $4.843 e-7$ & $7.4934 e-7$ & $9.7891 e-7$ & $7.5518 e-7$ \\
\hline
\end{tabular}

(i) Let $M=A, N=0$ and denote the method corresponding to Method 1 as $N M I$ and the method corresponding to Method 2 as $M I$, respectively.

(ii) Let $M=(1 / a) \widehat{D}-L$ and $N=(1 / \alpha-1) \widehat{D}+U$, where $\widehat{D},-L,-U$ are the diagonal, the strictly lower triangle, and the strictly upper triangle of the matrix $A$, respectively, and $\alpha$ is a relaxation parameter. Denote the methods corresponding to Method 1 as $\mathrm{NMSOR}_{\alpha}$ and Method 2 as $M S O R_{\alpha}$, respectively.
We first test the effect of the choice of matrix $\Omega$ in Method 1 on Problem 1. We let $\Omega=I, 2 I, 3 I, 4 I, 5 I$ and fix the dimension of the problem $n=400$. The results are listed in Tables 1 and 2. The column of the table has the following meaning.

Table 1 is the numerical result for NMI, and Table 2 is the numerical result for $\mathrm{NMSOR}_{\alpha}$ with fixed $\alpha=0.6$. As we can see from the tables, $\Omega$ has a great influence on the behaviour of the proposed method. In the following experiments, we fix 
$\Omega=4 I$. We compared our Method 1 with Method 2 proposed in [24]. The results are listed in Tables 3-6. “-” means the method failed to find the solution. From the tables, we can see that our method outperforms the method proposed in [24] regardless of the number of iterations or the CPU elapsed time. In these two examples, NMI seems to be competitive with NMSOR with the best parameters. In addition, as we can see from Tables 4 and 6, methods NMI and NMSOR may be independent of the dimensions.

\section{Conclusions}

In this paper, we have presented an efficient class of modulus-based matrix splitting methods for NCPs, which are based on an implicit system of fixed-point equations. A number of sufficient conditions to guarantee the convergence of the novel iteration method are presented. The proposed method is easy to implement, and in each iteration, there is no need to solve the nonlinear equations or linear complementarity subproblems; hence, the calculation cost is small. The comparisons of the numerical results show the computational efficiency of the proposed method. There are still many studies that need to be done. For example, we can investigate the two-step modulus-based matrix splitting method, that is, in each iteration, the method implements a forward sweep followed by a backward sweep. We can expect the two-step method to achieve a higher computing efficiency. Moreover, we can apply this new method to other complementarity problems, such as the implicit complementarity problems, and discuss its convergence (Table 5).

\section{Data Availability}

All the datasets used in this paper are available from the corresponding author upon request.

\section{Conflicts of Interest}

The authors declare that there are no conflicts of interest regarding the publication of this article.

\section{References}

[1] C. M. Elliott and J. R. Ockendon, "Weak and variational methods for moving boundary problems," Research Notes in Mathematics, Vol. 59, Pitman, London, UK, 1982.

[2] G. H. Meyer, "Free boundary problems with nonlinear source terms," Numerische Mathematik, vol. 43, no. 3, pp. 463-483, 1984.

[3] J. F. Rodrigues, Obstacle Problems in Mathematical Physics, Elsevier Science Publishers B.V., Amsterdam, Netherlands, 1987.

[4] Z. Luo, L. Qi, and N. Xiu, "The sparsest solutions to Z-tensor complementarity problems," Optimization Letters, vol. 11, no. 3, pp. 471-482, 2017.

[5] F. Wen, J. Shi, and Z. Zhang, "Closed-form estimation algorithm for EMVS-MIMO radar with arbitrary sensor geometry," Signal Processing, vol. 186, Article ID 108117, 2021.

[6] T. Shu, J. He, and V. Dakulagi, "3-D near-field source localization using a spatially spread acoustic vector sensor,"
IEEE Transactions on Aerospace and Electronic Systems, p. 1, 2021.

[7] T. Shu, J. He, and L. Li, "Near-field passive localization and gain-phase compensation with partly calibrated arrays," IEEE Transactions on Aerospace and Electronic Systems, p. 1, 2021.

[8] F. Facchinei and J.-S. Pang, Finite-dimensional Variational Inequalities and Complementarity Problems, Springer Science and Business Media, New York, NY, USA, 2003.

[9] Y. Zhang, "Multilevel projection algorithm for solving obstacle problems," Computers \& Mathematics with Applications, vol. 41, no. 12, pp. 1505-1513, 2001.

[10] L. Badea, X.-C. Tai, and J. Wang, "Convergence rate analysis of a multiplicative Schwarz method for variational inequalities," SIAM Journal on Numerical Analysis, vol. 41, no. 3, pp. 1052-1073, 2003.

[11] Y. Jiang and J. Zeng, "A multiplicative Schwarz algorithm for the nonlinear complementarity problem with an $M$-function," Bulletin of the Australian Mathematical Society, vol. 82, no. 3, pp. 353-366, 2010.

[12] J.-X. Zhao and S. Wang, "A power penalty approach to a discretized obstacle problem with nonlinear constraints," Optimization Letters, vol. 13, no. 7, pp. 1483-1504, 2019.

[13] S. Wang, "An interior penalty method for a large-scale finitedimensional nonlinear double obstacle problem," Applied Mathematical Modelling, vol. 58, pp. 217-228, 2018.

[14] Y. Duan, S. Wang, and Y. Zhou, "A power penalty approach to a mixed quasilinear elliptic complementarity problem," Journal of Global Optimization, vol. 81, pp. 901-918, 2021, https://doi.org/10.1007/s10898-021-01000-7.

[15] Z. Sun and J. Zeng, "A monotone semismooth Newton type method for a class of complementarity problems," Journal of Computational and Applied Mathematics, vol. 235, no. 5, pp. 1261-1274, 2011.

[16] Z.-Z. Bai and D. J. Evans, "Matrix multisplitting relaxation methods for linear complementarity problems," International Journal of Computer Mathematics, vol. 63, no. 3-4, pp. 309-326, 1997.

[17] Z.-Z. Bai, "On the monotone convergence of matrix multisplitting relaxation methods for the linear complementarity problem," IMA Journal of Numerical Analysis, vol. 18, no. 4, pp. 509-518, 1998.

[18] Z.-Z. Bai, "On the convergence of the multisplitting methods for the linear complementarity problem," SIAM Journal on Matrix Analysis and Applications, vol. 21, no. 1, pp. 67-78, 1999.

[19] Z. Z. Bai and D. J. Evans, "Matrix multisplitting methods with applications to linear complementarity problems: parallel synchronous and chaotic methods," Calculateurs Paralleles Reseaux et systemes repartis, vol. 13, pp. 125-154, 2001.

[20] Z.-Z. Bai, "Modulus-based matrix splitting iteration methods for linear complementarity problems," Numerical Linear Algebra with Applications, vol. 17, no. 6, pp. 917-933, 2010.

[21] W.-W. Xu and H. Liu, "A modified general modulus-based matrix splitting method for linear complementarity problems of H-matrices," Linear Algebra and Its Applications, vol. 458, pp. 626-637, 2014.

[22] W. Li, "A general modulus-based matrix splitting method for linear complementarity problems of $H$-matrices," Applied Mathematics Letters, vol. 26, no. 12, pp. 1159-1164, 2013.

[23] H. Zheng and W. Li, "The modulus-based nonsmooth Newton's method for solving linear complementarity problems," Journal of Computational and Applied Mathematics, vol. 288, pp. 116-126, 2015. 
[24] S.-L. Xie, H.-R. Xu, and J.-P. Zeng, “Two-step modulus-based matrix splitting iteration method for a class of nonlinear complementarity problems," Linear Algebra and Its Applications, vol. 494, pp. 1-10, 2016.

[25] Z. Xia and C. Li, "Modulus-based matrix splitting iteration methods for a class of nonlinear complementarity problem," Applied Mathematics and Computation, vol. 271, pp. 34-42, 2015.

[26] S. Wu and C. Li, "A class of new modulus-based matrix splitting methods for linear complementarity problem," Optimization Letters, 2021, https://doi.org/10.1007/s11590021-01781-6.

[27] Z.-Z. Bai and L.-L. Zhang, "Modulus-based synchronous twostage multisplitting iteration methods for linear complementarity problems," Numerical Algorithms, vol. 62, no. 1, pp. 59-77, 2013.

[28] A. Frommer and G. Mayer, "Convergence of relaxed parallel multisplitting methods," Linear Algebra and Its Applications, vol. 119, pp. 141-152, 1989. 